\title{
Z Source Inverter Topologies-A Survey
}

\author{
V. Saravanan ${ }^{* 1}$, M. Aravindan ${ }^{2}$, V. Balaji ${ }^{3}$, M. Arumugam ${ }^{4}$ \\ ${ }^{1,4}$ Arunai Engineering College, Tiruvannamalai, Tamilnadu, India \\ ${ }^{2}$ SCSVMV University \& TANGEDCO, Tamilnadu, India \\ ${ }^{3}$ School of Electrical Engineering, Bahir Dar University, Ethiopia \\ ${ }^{*}$ Corresponding author, e-mail: vsaranaec@yahoo.co.in
}

\begin{abstract}
Need for alternative energy sources to satisfy the rising demand in energy consumption elicited the research in the area of power converters/inverters. An increasing interest of using $Z$ source inverter/converter in power generation involving renewable energy sources like wind and solar energy for both off grid and grid tied schemes were originated from 2003. This paper surveys the literature of $Z$ source inverters/converter topologies that were developed over the years.
\end{abstract}

Keywords: Z source converter/inverter, topology modifications, voltage boosting

\section{Introduction}

Ever since the evolution of $Z$ source inverter, it has been an area of wide research especially due to its application in power generation based on various renewable energy sources [1-7]. Typical Z source inverter (ZSI) uses a LC impedance network between the source and voltage source inverter (VSI). Z source inverter (ZSI) has the property of stepping down or stepping up the input voltage, as a result, the output can be either higher or lower than the input voltage as per requirement. Moreover the conversion can be done in a single stage unlike the already existing systems with two stages of conversion.

Exhaustive researchs are carried out to compare the numerous characteristics of ZSI with the voltage source and current source inverters and explaining the generalized cascading concepts. This concept highly enhances the reliability of the inverter and is mostly achieved using the shoot through process [8-15]. Developments are also made in increasing the applicability in various other fields like electric vehicles, motor drives, power quality improvements and various high power applications. Studies are also conducted to determine the value of $Z$ source network based on the various requirements [16-23].

\section{Z source Inverter/ConverterTopologies}

Various new topologies have been developed in the $Z$ source inverter/converter primarily to improve the efficiency by reducing the number of switches, switching cycles and passive component, improving current and voltage gain and reducing component stresses [2445]. Various control strategies and topologies are developed to improve reliability [49-56] and reduce harmonics and other electromagnetic noise interference are found in [46-50].

Many improvements are made in the basic topology of ZSI to arrive at numerous topologies like switched inductor/capacitor, tapped inductor, magnetically coupled ZSI and transformer isolated type which are developed mainly to enhance the output voltage boosting and inversion ability along with reducing component stresses [31], [41], [65-81]. Other than three phase ZSI, single phase ZSI have been developed over these years with dual grounding, reduced switch and various control techniques [57-64]. Albeit the concept of $Z$ source power conversion introduced originally for dc-ac conversions, researchers are able to use the concept in developing a variety of converters which does dc-dc [82-87], ac-dc [88-90] and ac-ac conversions as well [90-130]. The ac-ac conversion also includes the integration of $Z$ source network in matrix converters including sparse matrix converters. These matrix converters are highly efficient with extended output voltage and the provision to control input power factor. They can also be used in application requiring bidirectional power flow [104-128]. In embedded 
ZSI topologies, the DC source is embedded within the Z network unlike the traditional one. Smoother and smaller current and voltage are maintained across the DC input source thus reducing the capacitor voltage stress and ripples in the input current with high boost ratio [131-136].

Quasi Z source inverters/converters topologies are developed as discussed in [42], [53-54], [63], [137-160]. Different types of these models are developed by just adding inductors, capacitors and diodes to the traditional topology to form switched inductor type, two stage network type etc. These new topologies have increased voltage gain, continuous and constant input current with reduced switching count, voltage and current stress. On top of it all, it requires lesser shoot through current for same voltage gains [161-180]. The quasi $Z$ source network is also used in implementing DC-DC converters as well [181-182].

$\mathrm{Z}$ source multi level inverters (MLI) are mainly a mixture of cascaded basic units and $\mathrm{H}$ bridge circuit, diode clamped. These units produce positive and zero voltage levels and the same time, it obtains positive, zero and negative voltage levels from $\mathrm{H}$ bridge circuit, so the number of semi conductor switches is reduced with respect to traditional multilevel inverters [182-190]. It is most reliable against short circuit faults and THD of injected voltage is decreased compared to traditional MLI. Single Z source based MLI with reduced number of switches is developed mainly for lower and medium power level application. Other ZSMLI topology like, single $Z$ source based cascaded transformer MLI uses the same technique as that of the traditional transformer based cascaded inverter, found to be more reliable against short circuit faults and also seemed to maintain the THD almost constant for different boost ratio.

With further developments to the concept of multi level ZSI Neutral Point Clamped (NPC) inverters with Z-source network became an attractive solution. Multi-level output is obtained with reduced passive components by connecting low cost front end diode rectifier to NPC ZSI. Concepts of quasi Z source and trans Z source inverters are introduced in NPC to obtain very high quality output voltage with lesser voltage distortions, reduced inverter noise and enhanced buck-boost features. Various modulation techniques like Pulse Width Modulation (PWM), space vector and digital controls like FPGA could be used effectively in NPC ZSI as well [192-206].

Trans $Z$ source neutral clamped inverter is developed by using a transformer and a capacitor to constitute the $\mathbf{Z}$ network. It is able to produce multi level output along with reduced passive components and improved the efficiency compared to traditional one. Increased voltage gain, reduced voltage stress, continuous input current and boost inversion capability are some of the features developed in Trans ZSI during these years [207-213]. As part of reducing the losses and improving the efficiency, a new topology called nine switch inverter is developed [214-217]. Even dual output could be obtained from these types of inverters. They are also able to provide bidirectional flow of power and find applications in hybrid electric vehicles [218-219]. In the concept of semi-ZSI only two active switches are used to achieve the same output with the special feature of no shoot through zero state as that of traditional ZSI [220-221].

Inverse Watkins-Johnson Topology is a robust one and has high immunity towards electromagnetic interference noise by allowing shoot-through of the inverter leg switches. As a modification of the transZSI LCCT ZSI is developed by integration of q-ZSI with a built in high frequency transformer and features continuous input current and improved relation between boost ratio to modulation index. It has more voltage gain compared to $q$-ZSI and prevents transformer core saturation compared to trans ZSI due to presence of two built-in DC-currentblocking capacitors connected in series with transformer windings [222-224].

Other topologies are developed mainly by altering the shape of the Z-network [225228]. $\Gamma$-source inverters have impedance network in $\Gamma$ shape with lesser passive components and boosted output voltage [229-230].

\section{Conclusion}

It is well understood from the recent researches that $Z$ source inverter/converter is gaining popularity and with advanced modulation techniques coming into play, it can be used in a wide range of applications. It can improve the efficiency of the drive systems, reduce harmonics and help in maintaining the power quality. Even years after its introduction, the scope of improvement and applicability in various applications are still high and still a hot topic for most of the researchers. With the need for change over from conventional sources of energy to new

Bulletin of EEI Vol. 6, No. 1, March 2017: 1-12 
renewable sources of energy in most of the applications, the importance of $Z$ source inverter/converter keeps rising day by day.

\section{Acknowledgements}

This work is part of the Central Power Research Institute Project, A Government of India Society, Ministry of Power, Bangalore (Project Code: RSOP/2015/DG/6/15122015). The support of this organization is gratefully acknowledged. The authors thank the management of Arunai Engineering College, Tiruvannamalai for providing the opportunity and facilities to do this work. The author, M. Aravindan, Assistant Executive Engineer-GIS, TANGEDCO, Tiruvannamalai like to thank the authorities of TANGEDCO, SCSVMV University, Kancheepuram, Bahir Dar University, Etopia and Arunai Engineering College, Tiruvannamalai. The authors thank Amal Thukku, Arunai Engineering College, Tiruvannamalai for his contribution.

\section{References}

[1] Dmitri Vinnikov, Andrii Chub, Elizaveta Liivik, Indrek Roasto. High-Performance Quasi-Z-Source Series Resonant DC-DC Converter for Photovoltaic Module-Level Power Electronics Applications. IEEE Transactions on Power Electronics. 2017; 32(5): 3634-3650.

[2] Yushan Liu, Haitham Abu-Rub, Baoming Ge. Front-End Isolated Quasi-Z-Source DC-DC Converter Modules in Series for High-Power Photovoltaic Systems-Part I: Configuration, Operation, and Evaluation. IEEE Transactions on Industrial Electronics. 2017; 64(1): 347-358.

[3] Yushan Liu, Haitham Abu-Rub, Baoming Ge. Front-End Isolated Quasi-Z-Source DC-DC Converter Modules in Series for High-Power Photovoltaic Systems-Part II: Control, Dynamic Model, and Downscaled Verification. IEEE Transactions on Industrial Electronics; 64(1): 359-368.

[4] Omar Ellabban, Haitham Abu-Rub. Z-Source Inverter: Topology Improvements Review. IEEE Industrial Electronics Magazine. 2016; 10(1): 6 -24.

[5] Chub A, Vinnikov D, Blaabjerg F, Peng FZ. A Review of Galvanically Isolated Impedance-Source DC-DC Converters. IEEE Transactions on Power Electronics. 2016; 31(4): 2808-2828.

[6] Yam P Siwakoti, Fang Zheng Peng, Frede Blaabjerg, Poh Chiang Loh, Graham E. Town. Impedance-Source Networks for Electric Power Conversion Part I: A Topological Review. IEEE Transactions on Power Electronics. 2015; 30(2): 699-716.

[7] Yam P Siwakoti, Fang Zheng Peng,Frede Blaabjerg, Poh Chiang Loh, Graham E. Town, Shuitao Yang. Impedance-Source Networks for Electric Power Conversion Part II: Review of Control and Modulation Techniques. IEEE Transactions on Power Electronics. 2015; 30(4): 1887-1906.

[8] Fang Zheng Peng. Z Source inverter. IEEE Transactions on Industry Applications. 2003; 39(2): 504-510.

[9] Ding Li, Feng Gao, Poh Chiang Loh, Miao Zhu, Frede Blaabjerg. Hybrid Source Impedance Networks: Layouts and Generalized Cascading Concepts. IEEE Transactions on Power Electronics. $2011 ; 26(7): 2028-2040$.

[10] Loh PC, Vilathgamuwa DM, Gajanayake CJ, Wong LT, Ang CP. Z source current type inverters: Digital Modulation and Logic Implementation. IEEE Transactions on Power Electronics. 2007; 22(1): 169-177.

[11] Gokhan Sen, Malik E Elbuluk. Voltage and current programmed modes in control of the $Z$ source converter. IEEE Transactions on Industry Applications. 2010; 46(2): 680-686.

[12] Peng FZ. Recent advances and applications of power electronics and motor drives power converters. IEEE ${ }^{34}$ th Annual Conference of Industrial Electronics (IECON). 2008: 30-33.

[13] Liu J, Jiang S, Cao D, Peng FZ. A digital current control of quasi Z source inverter with battery. IEEE Transactions on Industrial Informatics. 2013; 9(2): 928-937.

[14] Poh Chiang Loh, Ding Li, Frede Blaabjerg.Г-Z-Source Inverters. IEEE Transactions on Power Electronics. 2013; 28(11): 4880-4885.

[15] Rajakaruna S, Jayawickrama L. Steady state analysis and designing impedance network of Z source inverters. IEEE Transactions on Industrial Electronics. 2010; 57(7): 2483-2491.

[16] Jiudong Ding, Shaojun Xie, Yu Tang. Optimal design of the inductor in Z source inverter with single phase shoot through SVPWM Strategy. Energy Conversion Congress and Exposition. 2011: 28782882.

[17] Mo Wei, Loh Poh Chiang, Ding Yi.Optimal design of two level inverters. Conference Proceedings of IPEC. 2010: 705-710.

[18] Haiping Xu, Peng FZ, Lihua Chen, Xuhui Wen. Analysis and design of Bi directional Z source inverter for electrical vehicles. Twenty Third Annual IEEE Conference on Applied Power Electronics Conference and Exposition (APEC). 2008: 1252-1257. 
[19] Jin Wang. Practical design considerations of power electronics in hybrid and fuel cell vehicles. Vehicle Power and Propulsion Conference (VPPC). 2008; 1-6.

[20] Yifan Yu, Qianfan Zhang, Xiaofei Liu, Shumei Cui. DC link voltage ripple analysis and impedance network design of single phase $Z$ source inverter. ${ }^{14}$ th European Conference on Power Electronics and Applications. 2011: 1-10.

[21] Calzo GL, Lidozzi A, Solero L, Crescimbini F. Three phase Z source power supply design. IET Power Electronics. 2012; 5(8): 1270-1281.

[22] Lo Calzo G, Lidozzi A, Solero L, Crescimbini F. Three phase Z source power supply design and dynamic modeling. IEEE Energy Conversion Congress and Exposition (ECCE). 2011: 1339-1345.

[23] Rajesh K, A Amar Dutt, M Nandakumar. Modified Z source single phase two switch inverter. Proceedings of International Conference on Signal Processing, Communication, Computing and Networking Technologies (ICSCCN). 2011: 389-394.

[24] Poh Chiang Loh, Na Duan, Chao Liang, Feng Gao, Blaabjerg F. Z Source B4 Inverters. IEEE Power Electronics Specialists Conference (PESC). 2007: 1363-1369.

[25] Wei Mo, Poh Chiang Loh, Frede Blaabjerg. Asymmetrical $\Gamma$-Source Inverters. IEEE Transactions on Industrial Electronics. 2014; 61(2): 637-647.

[26] Antal R, Muntean N, Boldea I, Blaabjerg F. Novel, four switch, Z source three phase inverter. ${ }^{36}$ th Annual Conference on IEEE Industrial Electronics Society. 2010: 619-624.

[27] Banaei MR, Dehghanzadeh AR, Salary E, Khounjahan H, Alizadeh R. Z source based multilevel inverter with reduction of switches. IET Power Electronics.2012; 5(3): 385-392.

[28] Yu Tang, Shaojun Xie, Chaohua Zhang, Zegang Xu. Improved Z source inverter with reduced Z source capacitor voltage stress and soft start capability. IEEE Transactions on Power Electronics. 2009; 24(2): 409-415.

[29] Wei Qian, Fang Zheng Peng, Honnyong Cha. Trans-Z-source inverters. International Power Electronics Conference (IPEC). 2010: 1874-1881.

[30] Li Ding, Loh Poh Chiang, Zhu Miao, Gao Fen, Blaabjerg Frede. Cascaded switched inductor and tapped inductor $Z$ source inverters. Twenty-Sixth Annual IEEE Applied Power Electronics Conference and Exposition (APEC). 2011: 1661-1666.

[31] Poh Chiang Loh, Frede Blaabjerg. Magnetically coupled impedance source inverters. IEEE Transactions on Industry Applications. 2013; 49(5): 2177-2187.

[32] Kun Yu, Fang Lin Luo, Miao Zhu.Study of an improved Z source inverter: Small signal analysis. IEEE Conference on Industrial Electronics and Applications (ICIEA). 2010: 2169-2174.

[33] Rostami H, Khaburi DA. A new method for minimizing of voltage stress across devices in $Z$ source inverter. ${ }^{2}$ nd Power Electronics, Drive Systems and Technologies Conference (PEDSTC). 2011: 610-614.

[34] Minh Khai Nguyen, Young Cheol Lim, Yi Gon Kim. T Z Source Inverters. IEEE Transactions on Industrial Electronics. 2013; 60(12): 5686-5695.

[35] Najmi ES, Heydari M, Mohamadian M, Dehghan SM. Z source three phase four switch inverter with DC link split capacitor and comprehensive investigation of $Z$ source three phase four switch inverters. $3^{\text {rd }}$ Power Electronics and Drive Systems Technology (PEDSTC). 2012: 25-31.

[36] Yu Tang, Shaojun Xie, Chaohua Zhang. New structure and topological derivation of $Z$ source converters. ${ }^{4}$ th IEEE Conference Industrial Electronics and Applications (ICIEA). 2009: 788-793.

[37] Ding Li, Poh Chiang Loh, Miao Zhu, Feng Gao, Frede Blaabjerg. Enhanced boost Z source inverters with alternate cascaded switched and tapped inductor cells. IEEE Transactions on Industrial Electronics. 2013; 60(9): 3567-3578.

[38] Qian W, Peng FZ, Cha H. Trans Z source inverters. IEEE Transactions on Power Electronics. 2011; 26(12): 3453-3463.

[39] Marek Adamowicz, Jarosław Guzinski, Dmitri Vinnikov, Natalia Strzelecka.Trans Z source like inverter with built in DC current blocking capacitors. IEEE Conference-Workshop Compatibility and Power Electronics. 2011: 137-143.

[40] Husev O, Roncero Clemente C, Romero Cadaval, E, Vinnikov, D, Stepenko, S.Single phase three level neutral point clamped quasi Z source inverter. IET Power Electronics. 2015; 8(1): 1-10.

[41] Miao Zhu, Ding Li, Feng Gao, Poh Chiang Loh, Frede Blaabjerg. Extended topologies of tapped inductor $Z$ source inverters. ${ }^{8}$ th International Conference on Power Electronics-ECCE Asia. 2011: 1599-1605.

[42] Vinnikov D, Roasto I, Jalakas T. Comparative study of capacitor assisted extended boost qZSIs operating in continuous conduction mode. ${ }^{12}$ th Biennial Baltic Electronics Conference (BEC). 2010: 297-300.

[43] Nguyen MK, Lim YC, Kim Y. T Z Source Inverters. IEEE Transactions on Industrial Electronics. 2013; 60(12): 5686-5695.

[44] Husev Oleksandr Stepenko, Serhii Roncero Clemente, Carlos Romero Cadaval, Enrique, Vinnikov Dmitri. Single phase three level quasi $Z$ source inverter with a new boost modulation technique. ${ }^{38}$ th Annual Conference on IEEE Industrial Electronics Society (IECON).2012: 5852-5857. 
[45] Wei Mo, Poh Chiang Loh, Blaabjerg F, Peng Wang. Trans Z source and $\Gamma Z$ source neutral point clamped inverters. IET Power Electronics. 2015; 8(3): 371-377.

[46] Seung Yeol Oh, Young Gook Jung, Young Cheol Lim, Dea Kyong Kim, Sung Geun Song. Three phase $Z$ source hybrid active power filter system. International Conference on Electrical Machines and Systems (ICEMS). 2010: 391-396.

[47] Yifan Yu, Qianfan Zhang, Bin Liang, Shumei Cui. Single phase Z source inverter: Analysis and Low frequency harmonics elimination pulse width modulation. IEEE Energy Conversion Congress and Exposition (ECCE). 2011: 2260-2267.

[48] Ding Xinping, Liu Jian, Lu Yan, Yu Bo. A Novel Adjustable Speed System Based on Z Source Inverter. Proceedings of the 30th Chinese Control Conference. 2011: 3523-3527.

[49] BJ Rabi, R Arumugam. Harmonics study and comparison of Z Source Inverter with traditional Inverters. American Journal of Applied Sciences. 2015: 1418-1426.

[50] Das A, Chowdhury SP, Domijan A. Performance analysis of Z source inverter based ASD system with reduced harmonics. IEEE Conference on Power and Energy Society General MeetingConversion and Delivery of Electrical Energy in the $21^{\text {st }}$ Century. 2008: 1-7.

[51] Xupeng Fang, Zhiqiao Chen. Current fed Z source matrix converter. International Conference on Applied Superconductivity and Electromagnetic Devices (ASEMD). 2009: 93-96.

[52] Yaosuo Xue, Baoming Ge, Fang Zheng Peng. Reliability, efficiency, and cost comparisons of MW scale photovoltaic inverters. IEEE Energy Conversion Congress and Exposition (ECCE). 2012: 1627-1634.

[53] Selvia SK, Deepak FXE. Solar integrated current fed quasi Z source inverter with power buck boost capability. International Conference on Advances in Engineering, Science and Management (ICAESM). 2012: 190-195.

[54] Vinnikov D, Roasto I, Strzelecki R, Adamowicz M. Performance improvement method for the voltage-fed qZSI with continuous input current. ${ }^{15}$ th IEEE Mediterranean Electro technical Conference (MELECON) .2010: 1459-1464.

[55] Zhou Y, Liu L, Li H. A High-Performance Photovoltaic Module-Integrated Converter (MIC) Based on Cascaded Quasi Z Source Inverters (qZSI) Using eGaN FETs. IEEE Transactions on Power Electronics. 2013; 28(6): 2727-2738.

[56] Minh Khai Nguyen, Young-Cheol Lim, Sung-Jun Park. Improved Trans-Z-Source Inverter With Continuous Input Current and Boost Inversion Capability. IEEE Transactions on Power Electronics. 2013; 28(10): 4500-4510.

[57] Yu Tang, Shaojun Xie, Chaohua Zhang.Single phase Z source Inverter. IEEE Transactions on Power Electronics. 2011; 26(12): 3869-3873.

[58] Chandrashekhar T, Veerachary M. Control of single phase $Z$ source inverter for a grid connected system. International Conference on Power Systems (ICPS). 2009: 1-6.

[59] Thelukuntla CS, Veerachary M. Resonant controller based single-phase Z source inverter with LCL filter. Joint International Conference on Power Electronics, Drives and Energy Systems (PEDES). 2010: 1-6.

[60] Yi Zhao, Xiangning He, Z. John Shen, Liming Liu, Hui Li. $1 \mathrm{MHz}$ Cascaded Z source inverters for scalable grid interactive photovoltaic (PV) applications Using GaN Device.Energy Conversion Congress and Exposition. 2011: 2738-2745.

[61] Antal R, Muntean N, Boldea I. Modified Z source single phase inverter for single phase PM synchronous motor drives. ${ }^{11}$ th International Conference on Optimization of Electrical and Electronic Equipment (OPTIM 2008). 2008: 245-250.

[62] Boldea I, Antal R, Muntean N. Modified Z source single phase inverter with two switches. International Symposium on Industrial Electronics. 2008: 257-263.

[63] TW Chun, HH Lee, HG Kim, EC Nho. Power control for a PV generation system using a single phase grid connected quasi $Z$ source inverter. ${ }^{8}$ th International Conference on Power ElectronicsECCE Asia. 2011: 889-893.

[64] Long Huang, Ming Zhang, Lijun Hang, Wenxi Yao, Zhengyu Lu. A family of three switches three state single phase Z source inverters. IEEE Transactions on Power Electronics. 2013; 28(5): 23172329.

[65] Ding Li, Poh Chiang Loh, Miao Zhu, Feng Gao, Frede Blaabjerg. Generalized multicell switched inductor and switched capacitor Z source inverters. IEEE Transactions on Power Electronics. 2013; 28(2): 837-848.

[66] Upadhyay S, Adda R, Mishra S, Joshi A. A switched-boost topology for renewable power application. Conference Proceedings of IPEC. 2010: 758-762.

[67] Miao Zhu, Kun Yu, Fang Lin Luo. Switched Inductor Z Source Inverter. IEEE Transactions on Power Electronics. 2010; 25(8): 2150-2158.

[68] Miao Zhu, Kun Yu, Fang Lin Luo. Topology analysis of a switched inductor Z source inverter. IEEE Conference on Industrial Electronics and Applications (ICIEA). 2010: 364-369.

[69] Yamashita N, Asano M, Yamanaka M, Koizumi H. A-Z source inverter accepting rapid reverse current flow. IEEE Region 10 Conference TENCON. 2010: 48-53. 
[70] Quoc Nam Trinh, Hong Hee Lee, Tae Won Chun. A New Z source inverter topology to improve voltage boost ability. ${ }^{8}$ th International Conference on Power Electronics-ECCE Asia. 2011: 19811986.

[71] Miao Zhu, Ding Li, Poh Chiang Loh, Blaabjerg F. Tapped inductor Z source inverters with enhanced voltage boost inversion abilities. 2010 IEEE International Conference Sustainable Energy Technologies (ICSET). 2010: 1-6.

[72] Yu Tang, Shaojun Xie, Chaohua Zhang. An Improved Z-Source Inverter. IEEE Transactions on Power Electronics. 2011; 26(12): 3865-3868.

[73] Ferraz PEP, Bradaschia F, Cavalcanti MC, Neves FAS, Azevedo GMS. A modified Z-source inverter topology for stable operation of transformerless photovoltaic systems with reduced leakage currents. Brazilian Power Electronics Conference (COBEP). 2011: 615-622.

[74] Adda R, Mishra S, Joshi A. Analysis and PWM Control of Switched Boost Inverter. IEEE Transactions on Industrial Electronics. 2013; 60(12): 5593-5602.

[75] Upadhyay S, Adda R, Mishra S, Joshi A. Derivation and characterization of switched boost inverter. ${ }^{14}$ th European Conference on Power Electronics and Applications (EPE). 2011: 1-10.

[76] Ismeil M A, Kouzou A, Kennel R, Abu-Rub H, Orabi M. A new switched inductor quasi $Z$ source inverter topology. ${ }^{15}$ th International Power Electronics and Motion Control Conference (EPE/PEMC). 2012: 1-6.

[77] Poh Chiang Loh, Blaabjerg, F. Magnetically coupled impedance-source inverters. IEEE Energy Conversion Congress and Exposition (ECCE). 2012: 4545-4552.

[78] Li D, Loh P, Zhu M, Gao F, Blaabjerg F. Enhanced Boost Z Source Inverters with Alternate Cascaded Switched and Taped Inductor Cells. IEEE Transactions on Industrial Electronics. 2013; 60(99): 3567-3578.

[79] Shuai Jiang, Dong Cao, Peng FZ. High frequency transformer isolated Z-source inverters. Twenty Sixth Annual Applied Power Electronics Conference and Exposition (APEC). 2011: 442-449.

[80] X Ding, Z Qian, S Yang, B Cui, FZ Peng. A high-performance Z-source inverter operating with small inductor at wide-range load.Twenty Second Annual IEEE Applied Power Electronics Conference. 2007: 615-620.

[81] Dehghan S.M, Mohamadian M, Siefi E. Discontinuous energy pump source inverters. $2^{\text {nd }}$ Power Electronics Drive Systems and Technologies Conference (PEDSTC). 2011: 427-432.

[82] Xupeng Fang. A novel $Z$ source $d c-d c$ converter. IEEE International Conference on Industrial Technology (ICIT). 2008: 1-4.

[83] B Axelrod, Y Berkovich, A loinovici.Switched capacitor/switched inductor structures for getting transformer less hybrid dc-dc PWM converters. IEEE Transactions on Circuit and Systems Fundamental Theory Applications. 2008; 55(2): 687-696.

[84] Shindo Y, Yamanaka M, Koizumi H. Z source DC-DC converter with cascade switched capacitor. ${ }^{37}$ th Annual Conference on IEEE Industrial Electronics Society (IECON). 2011: 1665-1670.

[85] Asano M, Abe D, Koizumi H. A common grounded Z source buck boost converter. IEEE International Symposium on Circuits and Systems (ISCAS). 2011: 490-493.

[86] H Cha, FZ Peng, DW Yoo. Distributed impedance network (Z-network) dc-dc converter. IEEE Transactions on Power Electronics. 2010; 25(11): 2722-2733.

[87] Biao Zhao, Qingguang Yu, Zhiwei Leng, Xiyou Chen. Switched Z source isolated bidirectional DCDC converter and its phase shifting shoot-through bivariate coordinated control strategy. IEEE Transactions on Industrial Electronics.2012; 59(12): 4657-4670.

[88] Fan Zhang, Peng FZ, Zhaoming Qian. Z-H converter. Power Electronics Specialists Conference. 2008: 1004-1007.

[89] Hosseini S H, Sedaghati F, Sarhangzadeh M. Improved power quality three phase AC-DC converter. International Conference on Electrical Machines and Systems (ICEMS). 2010: 148-153.

[90] Xupeng Fang, Maoyong Cao, Zhiqiao Chen. Z source AC-DC-AC converter for mining applications. International Conference on Electrical Machines and Systems (ICEMS). 2010: 44-47.

[91] Xu Peng Fang, Zhao Ming Qian, Fang Zheng Peng. Single phase Z source PWM AC-AC converters. IEEE Power Electronics Letters. 2005; 3(4): 121-124.

[92] $Y$ Tang, SJ Xie, CH Zhang. Z-source AC-AC converters solving commutation problem. IEEE Transactions on Power Electronics. 2007; 22(6): 2146-2154.

[93] F Zhang, X Fang, FZ Peng, Z Qian. A new three phase AC-AC Z source converter. Annual IEEE Applied Power Electronics Conference Expo. 2006: 123-126.

[94] Von Zimmermann M, Labusch S, Piepenbreier B. Bi-directional AC-AC Z-source inverter with active rectifier and feed forward control.Energy Conversion Congress and Exposition (ECCE). 2010: 3180-3186.

[95] MK Nguyen, YG Jung, YC Lim. Single phase Z source ac-ac converter with wide range output voltage operation. Journal of Power Electronics. 2009; 9(5): 736-747.

[96] Uthirasamy R, Srimaheswaran V, Suganya C. Impedance source inverter based ac-ac converter system. International Conference on Sustainable Energy and Intelligent Systems (SEISCON). 2011: 207-212.

Bulletin of EEI Vol. 6, No. 1, March 2017: 1-12 
[97] Karaman E, Farasat M, Trzynadlowski AM. А ЗФ-ЗФ quasi Z-source matrix converter for residential wind energy systems. IEEE Energy Conversion Congress and Exposition (ECCE). 2012: 240-246.

[98] Karaman Ekrem, Farasat Mehdi, Trzynadlowski Andrzej M. Matrix converter with a series Z-source. ${ }^{38}$ th Annual Conference on IEEE Industrial Electronics Society (IECON). 2012: 6093-6098.

[99] Lechler M, Piepenbreier B. Regenerative SiC frequency converter with compact Z-source DC-link and sinusoidal output voltage. International Symposium on Power Electronics, Electrical Drives, Automation and Motion (SPEEDAM). 2012: 1506-1511.

[100] MK Nguyen, YG Jung, HY Yang, YC Lim.Single-phase Z-source cycloconverter with safecommutation strategy. IET Power Electronics. 2010; 3(2): 232-242.

[101] Hafiz Furqan Ahmed, Honnyong Cha, Ashraf Ali Khan, Heung Geun Kim. A Family of HighFrequency Isolated Single-Phase Z-Source AC-AC Converters with Safe-Commutation Strategy. IEEE Transactions on Power Electronics. 2016; 31(11): 7522 -7533.

[102] Khosravi F, Azli NA, Kaykhosravi A. A new single phase to three phase converter using Quasi Zsource network. IEEE Applied Power Electronics Colloquium (IAPEC). 2011: 46-50.

[103] X Fang, FZ Peng. Novel three phase current fed Z source ac ac converter. IEEE Power Electronics Specialists Conference. 2007: 2993-2996.

[104] Karaman E, Niu F, Trzynadlowski AM. Three phase switched inductor Z source matrix converter. Twenty-Seventh Annual IEEE Applied Power Electronics Conference and Exposition (APEC). 2012: 1449-1454.

[105] Omar Ellabban, Haitham Abu Rub, Sertac Bayhan. Z source matrix converter: An overview. IEEE Transactions on Power Electronics. 2016; 31(11): 7436-7450.

[106] Xiong Liu, Poh Chiang Loh, Fang Zheng Peng, Peng Wang, Feng Gao. Modulation of three-level Zsource indirect matrix converter. Energy Conversion Congress and Exposition (ECCE). 2010: 31953201.

[107] Shuo Liu, Baoming Ge, Xinjian Jiang, Abu-Rub, H, Fang Zheng Peng. Comparative evaluation of three $\mathrm{Z}$ source/quasi $\mathrm{Z}$ source indirect matrix converters. IEEE Transactions on Industrial Electronics.2015; 62(2): 692-701.

[108] Nguyen TD, Nguyen HM, Hong-Hee Lee, Tae-Won Chun. An approach of sparse matrix converter using Z-source network. International Power Electronics Conference (IPEC). 2010: 2774-2780.

[109] Xiong Liu, Poh Chiang Loh, Fang Zheng Peng, Peng Wang. Optimal modulation of indirect Z-source matrix converter. International Power Electronics Conference (IPEC). 2010; 3049-3056.

[110] Ekrem Karaman, Mehdi Farasat, Andrzej M Trzynadlowski. A comparative study of series and cascaded Z-source matrix converters. IEEE Transactions on Industrial Electronics. 2014; 61(10): 5164-5173.

[111] Kiwoo Park, Kyo-Beum Lee, Frede Blaabjerg. Improving output performance of a Z source sparse matrix converter under unbalanced input-voltage conditions. IEEE Transactions on Power Electronics. 2012; 27(4): 2043-2054.

[112] Weizhang Song, Yanru Zhong. A study of z-source matrix converter with high voltage transfer ratio. IEEE Vehicle Power and Propulsion Conference. 2008: 1-6.

[113] MK Nguyen, YG Jung, YC Lim, YM Kim. A single-phase Z-source buck-boost matrix converter. IEEE Transactions on Power Electronics. 25(2): 453-462.

[114] Baoming Ge, Qin Lei, Wei Qian, Fang Zheng Peng. A family of Z-source matrix converters. IEEE Transactions on Industrial Electronics. 2012; 59(1): 35-46.

[115] Fedyczak Z, Szczesniak P, Kaniewski J, Tadra G. Implementation of three-phase frequency converters based on PWM AC matrix reactance chopper with buck-boost topology.European Conference on Power Electronics and Applications (EPE). 2009: 1-10.

[116] Shuo Liu, Baoming Ge, Yushan Liu, Haitham Abu-Rub, Robert S. Balog, Hexu Sun. Modeling, Analysis, and Parameters Design of LC-Filter-Integrated Quasi Z Source Indirect Matrix Converter. IEEE Transactions on Power Electronics.2016; 31(11): 7544-7555.

[117] Qin Lei, Peng FZ, Baoming Ge. Pulse Width Amplitude Modulated voltage fed quasi-Z-source direct matrix converter with maximum constant boost. Twenty-Seventh Annual IEEE Applied Power Electronics Conference and Exposition (APEC). 2012: 641-646.

[118] Milan G, Mohamadian M, Seifi Najmi E, Dehghan SM. A single to three phase Z source matrix converter. ${ }^{3}$ rd Power Electronics and Drive Systems Technology (PEDSTC). 2012: 1-6.

[119] Kiwoo Park, Kyo Beum Lee, Frede Blaabjerg. Improving output performance of a Z-source sparse matrix converter under unbalanced Input voltage conditions. IEEE Transactions on Power Electronics. 2012; 27(4): 2043-2054.

[120] Xiong Liu, Poh Chiang Loh, Peng Wang, Xiaoqing Han. Improved Modulation Schemes for Indirect Z-source Matrix Converter with Sinusoidal Input and Output Waveforms. IEEE Transactions on Power Electronics. 2012; 27(9): 4039-4050.

[121] Weizhang Song, Yanru Zhong, Hao Zhang, Xiangdong Sun, Qi Zhang, Wei Wang. A study of Z Source dual bridge matrix converter immune to abnormal input voltage disturbance and with high voltage transfer ratio. IEEE Transactions on Industrial Informatics. 2013; 9(2): 828-838. 
[122] Keping You, Rahman FM. Modulation and Control Schemes for A New Power Converter Based on Z-source and Matrix Converter for ISA 42 V Power Net System. International Conference on Power Electronics and Drives Systems (PEDS). 2005: 436-441.

[123] Kiwoo Park, Eun-Sil Lee, Kyo-Beum Lee. A-Z source sparse matrix converter with a fuzzy logic controller based compensation method under abnormal input voltage conditions. IEEE International Symposium on Industrial Electronics (ISIE). 2010: 614-619.

[124] Keping You, Rahman MF. Application of General Space Vector Modulation Approach of AC-AC Matrix Converter Theory to A New Bidirectional Converter for ISA 42 V System. IEEE IAS Annual Meeting Industry Applications Conference. 2006: 2480-2487.

[125] Kiwoo Park, Eun-Sil Lee, Kyo-Beum Lee. A-Z-source sparse matrix converter with a fuzzy logic controller based compensation method under abnormal input voltage conditions. International Symposium on Industrial Electronics (ISIE). 2010: 614-619.

[126] You K, Rahman MF.Constructing A Novel Power Converter by Matrix Converter Theory and ZSource Inverter Concepts for ISA $42 \mathrm{~V}$ Power Net System. ${ }^{41}$ st IEEE Industry Applications Conference. 2006: 2101-2108.

[127] Keping You, Rahman MF. A Matrix Z-Source Converter with AC-DC Bidirectional Power Flow for an Integrated Starter Alternator System. IEEE Transactions on Industry Applications. 2009; 45(1): 239-248.

[128] Kiwoo Park, Kyo Beum Lee, Frede Blaabjerg. Improving Output Performance of a Z-source Sparse Matrix Converter under Unbalanced Input Voltage Conditions. IEEE Transactions on Power Electronics. 2012; 27(4): 2043-2054.

[129] Keping You, Rahman MF. A matrix-Z-source converter for automotive integrated starter alternator system. Twenty-Third Annual IEEE Applied Power Electronics Conference and Exposition (APEC). 2008: 273-279.

[130] Hosseini SH, Sedaghati F, Sarhangzadeh M. Modeling and simulation of a new single phase ac-ac converter. International Conference on Electrical and Electronics Engineering. 2009: 226-229.

[131] Gao F, Loh PC, Blaabjerg F, Gajanayake CJ. Operational analysis and comparative evaluation of embedded Z-Source inverters. IEEE Power Electronics Specialists Conference (PESC). 2008: 2757-2763.

[132] Poh Chiang Loh, Feng Gao, Blaabjerg F. Embedded EZ-Source Inverters. IEEE Transactions on Industry Applications. 2010; 46(1): 256-267.

[133] F Gao, PC Loh, D Li, F Blaabjerg. Asymmetrical and symmetrical embedded Z-source inverters. IET Power Electronics. 2011; 4(2): 181-193.

[134] Itozakura H, Koizumi H. Embedded Z Source inverter with switched inductor. ${ }^{37}$ th Annual Conference on IEEE Industrial Electronics Society (IECON). 2011: 1342-1347.

[135] Ebrahim Babaei, Elias Shokati Asl, Mohsen Hasan Babayi, Sara Laali. Developed embedded switched Z source inverter. IET Power Electronics. 2016; 9(9): 1828-1841.

[136] Mo Wei, Poh Chiang Loh, Frede Blaabjerg. Asymmetrical transformer based embedded Z-source inverters. IET Power Electronics. 2013; 6(2): 261-269.

[137] Shahparasti M, Sadeghi Larijani A, Fatemi A, Yazdian Varjani A, Mohammadian M. Quasi Z-source inverter for photovoltaic system connected to single phase AC grid. ${ }^{1}$ st Power Electronic \& Drive Systems \& Technologies Conference (PEDSTC). 2010: 456-460.

[138] Nguyen M, Lim Y, Cho G. Switched Inductor Quasi Z Source Inverter. IEEE Transactions on Power Electronics. 2011; 26(11): 3183-3191.

[139] Baoming Ge, Abu-Rub H, Fang Zheng Peng, Qin Lei, de Almeida AT, Ferreira FJTE, Dongsen Sun, Yushan Liu. An Energy Stored Quasi Z Source Inverter for Application to Photovoltaic Power System. IEEE Transactions on Industrial Electronics. 2013; 60(10): 4468-4481.

[140] Feng Guo, Lixing Fu, Chien-Hui Lin, Cong Li, Woong chul Choi, Jin Wang.Development of an 85 kW Bidirectional Quasi-Z-Source Inverter With DC Link Feed Forward Compensation for Electric Vehicle Applications. IEEE Transactions on Power Electronics. 2013; 28(12): 5477-5488.

[141] J Anderson, FZ Peng. A class of quasi Z source inverters. IEEE Industrial Applications Society Annual Meeting. 2008: 1-7.

[142] Anderson J, Peng FZ. Four quasi Z source inverters. Power Electronics Specialists Conference. 2008: 2743-2749.

[143] Nguyen MK, Lim YC, Choi JH. Two switched inductor quasi Z source inverters. IET Power Electronics. 2012; 5(7): 1017-1025.

[144] Yufei Zhou, Wenxin Huang, Jianwu Zhao, Ping Zhao. Tapped inductor quasi Z source inverter. Twenty Seventh Annual IEEE Applied Power Electronics Conference and Exposition (APEC). 2012: 1625-1630.

[145] Beer K, Piepenbreier B. Properties and advantages of the quasi-Z-source inverter for DC-AC conversion for electric vehicle applications. Emobility Electrical Power Train. 2010: 1-6.

[146] Yuan Li, Anderson J, Peng FZ, Dichen Liu. Quasi Z source inverter for photovoltaic power generation systems. Twenty-Fourth Annual IEEE Applied Power Electronics Conference and Exposition. 2009: 918-924.

Bulletin of EEI Vol. 6, No. 1, March 2017: 1-12 
[147] Yushan Liu, Baoming Ge, Ferreira FJTE, de Almeida AT, Abu-Rub H. Modeling and SVPWM control of quasi Z source inverter. ${ }^{11}$ th International Conference on Electrical Power Quality and Utilisation (EPQU). 2011: 1-7.

[148] Vinnikov D, Roasto I. Quasi Z Source based isolated DC/DC converters for distributed power generation. IEEE Transactions on Industrial Electronics. 2011; 58(1): 192-201.

[149] J Zakis, D Vinnikov, I Roasto, L Ribickis. Quasi Z source inverter based bidirectional DC/DC converter: Analysis of experimental results. ${ }^{7}$ th International Conference-Workshop Compatibility and Power Electronics (CPE). 2011: 394-399.

[150] Minh Khai Nguyen, Young Gook Jung, Young Cheol Lim, Duk Guen Cha. Single phase quasi Z source AC/AC converter. ${ }^{31}$ st International Telecommunications Energy Conference. 2009: 1-5.

[151] Vinnikov D, Roasto I, Strzelecki R, Adamowicz M. Two stage quasi Z source network based stepup $D C / D C$ converter. IEEE International Symposium on Industrial Electronics (ISIE). 2010: 1143-1148.

[152] Vinnikov D, Roasto I, Strzelecki R, Adamowicz M.Step-Up DC/DC Converters with Cascaded Quasi-Z-Source Network. IEEE Transactions on Industrial Electronics. 2012; 59(10): 3727-3736.

[153] Roasto I, Vinnikov D, Jalakas T Zakis J, Ott S. Experimental study of shoot-through control methods for qZSI-based DC/DC converters. International Symposium on Power Electronics Electrical Drives Automation and Motion (SPEEDAM). 2010: 29-34.

[154] Amal Thukku, V Saravanan, R Ramanujam, M Arumugam.Improved Quasi Z Source Inverter topologies for Photovoltaic Systems. Proceedings of the IET-Chennai Third International Conference on Sustainable Energy and Intelligent Systems-SEISCON. 2012: 219-224.

[155] Martinez C, Jalakas T, Vinnikov D, Lazaro A, Barrado A. QZSI DC/DC converters in input series output parallel connection for distributed generation. International Symposium on Power Electronics, Electrical Drives, Automation and Motion (SPEEDAM). 2012: 952-957.

[156] Siwakoti Yam P, Town Graham E. Performance of distributed DC power system using quasi ZSource Inverter based DC/DC converters. Twenty-Eighth Annual Applied Power Electronics Conference and Exposition (APEC). 2013: 1946 -1953.

[157] Yang S, Peng FZ, Lei Q, Inoshita R, Qian Z. Current fed Quasi Z Source inverter with voltage buck boost and regeneration capability. IEEE Transactions on Industry Applications. 2011; 47(2): 882892.

[158] Qin Lei, Fang Z Peng, Baoming Ge. Transient modeling of current fed Quasi Z source inverter. Energy Conversion Congress and Exposition. 2011: 2283-2287.

[159] Shuitao Yang, Qin Lei, Peng F Z, Inoshita R, Zhaoming Qian. Current fed quasi Z source inverter with coupled inductors. Energy Conversion Congress and Exposition (ECCE). 2009: 3683-3689.

[160] Huitao Yang, Peng FZ, Lei Q, Inoshita R, Zhaoming Qian. Current fed quasi Z source inverter with voltage buck boost and regeneration capability. Energy Conversion Congress and Exposition. 2009: 3675-3682.

[161] Yushan Liu, Abu Rub H, Baoming Ge. Z-Source/Quasi-Z-Source Inverters: Derived Networks, Modulations, Controls, and Emerging Applications to Photovoltaic Conversion. IEEE Industrial Electronics Magazine. 2014; 8(4): 32-44.

[162] Yuan Li, Shuai Jiang, Cintron Rivera JG, Fang Zheng Peng. Modeling and control of Quasi Z source inverter for distributed generation applications. IEEE Transactions on Industrial Electronics. 2013; 60(4): 1532-1541.

[163] Abu-Rub H, lqbal A, Moin Ahmed Sk, Peng FZ, Li Y, Baoming G. Quasi Z Source Inverter based photovoltaic generation system with maximum power tracking control using ANFIS. IEEE Transactions on Sustainable Energy. 2013; 4(1): 11- 20.

[164] Minh-Khai Nguyen, Young Cheol Lim, Yong Jae Kim. A modified single phase Quasi Z Source ACAC converter. IEEE Transactions on Power Electronics. 2012; 27(1): 201-210.

[165] Qin Lei, Dong Cao, Fang Zheng Peng. Novel loss and harmonic minimized vector modulation for a current fed quasi Z source inverter in HEV motor drive application. IEEE Transactions on Power Electronics. 2014; 29(3): 1344-1357.

[166] Minh Khai Nguyen, Tuan Vu Le, Sung Jun Park, Young Cheol Lim. A class of quasi-switched boost inverters. IEEE Transactions on Industrial Electronics. 2015; 62(3): 1526-1536.

[167] Liqiang Yang, Dongyuan Qiu, Bo Zhang, Guidong Zhang. High performance quasi-Z-source inverter with low capacitor voltage stress and small inductance. IET Power Electronics. 2015; 8(6): 10611067.

[168] Khajesalehi J, Sheshyekani K, Hamzeh M, Afjei E. High performance hybrid photovoltaic battery system based on quasi Z source inverter: application in micro grids. IET Generation, Transmission \& Distribution. 2015; 9(10): 895-902.

[169] Dongsen Sun, Baoming Ge, Weihua Liang, Abu-Rub H, Fang Zheng Peng. An energy stored quasiZ-source cascade multilevel inverter-based photovoltaic power generation system. IEEE Transactions on Industrial Electronics. 2015; 62(9): 5458-5467.

[170] Ahmed H, Cha H, Kim S, Kim H. Switched Coupled Inductor Quasi Z Source Inverter. IEEE Transactions on Power Electronics.2016; 31(2): 1241-1254. 
[171] Nguyen M, Lim Y, Park S. A comparison between single-phase quasi Z source and quasi switched boost inverters. IEEE Transactions on Industrial Electronics. 2015; 62(10): 6336-6344.

[172] Battiston A, Miliani E, Pierfederici S, Meibody Tabar F. A novel quasi Z source inverter topology with special coupled inductors for input current ripples cancellation. IEEE Transactions on Power Electronics. 2016; 31(3): 2409-2416.

[173] V Fernão Pires, Armando Cordeiro, Daniel Foito, Joao F Martins. Quasi-Z-source inverter with a Ttype converter in normal and failure mode. IEEE Transactions on Power Electronics. 2016; 31(11): 7462-7470.

[174] Yushan Liu, Baoming Ge, Haitham Abu-Rub, Hexu Sun, Fang Zheng Peng, Yaosuo Xue, .Model predictive direct power control for active power decoupled single-phase quasi-Z -source inverter. IEEE Transactions on Industrial Informatics. 2016; 12(4): 1550-1559.

[175] Thierry Kayiranga, Hongbo Li, Xinchun Lin, Yanjun Shi, Hui Li. Abnormal operation state analysis and control of asymmetric impedance network-based quasi-Z-source PV inverter (AIN-qZSI). IEEE Transactions on Power Electronics. 2016; 31(11): 7642-7650.

[176] Baoming Ge, Yushan Liu, Haitham Abu-Rub, Robert S. Balog, Fang Zheng Peng, Stephen McConnell, Xiao Li. Current ripple damping control to minimize impedance network for single-phase quasi-Z source inverter system. IEEE Transactions on Industrial Informatics. 2016; 12(3): 10431054.

[177] Baoming Ge, Yushan Liu, Haitham Abu Rub, Robert S Balog, Fang Zheng Peng, Hexu Sun, Xiao $\mathrm{Li}$. An active filter method to eliminate dc-side low-frequency power for a single-phase quasi-Zsource inverter. IEEE Transactions on Industrial Electronics. 2016; 63(8): 4838-4848.

[178] Yan Zhou, Hongbo Li, Hui Li. A single-phase PV quasi Z source inverter with reduced capacitance using modified modulation and double frequency ripple suppression control. IEEE Transactions on Power Electronics. 2016; 31(3): 2166-2173.

[179] Alexandre Battiston, El-Hadj Miliani, Serge Pierfederici, Farid Meibody Tabar. Efficiency improvement of a quasi-Z-source inverter-fed permanent-magnet synchronous machine-based electric vehicle. IEEE Transactions on Transportation Electrification. 2016; 2(1): 14-23.

[180] Mostafa Mosa, Robert S Balog, Haitham Abu Rub. High performance predictive control of quasi impedance source inverter. IEEE Transactions on Power Electronics. 2017; 32(4): 3251-3262.

[181] Minh Khai Nguyen, Young Cheol Lim, Joon Ho Choi, Geum Bae. Isolated high step-up dc-dc converter based on quasi-switched-boost network. IEEE Transactions on Industry Electronics. 2016; 63(12): 7553-7562.

[182] Husev O Liivik L, Blaabjerg F, Chub A, Vinnikov D, Roasto I. Galvanically Isolated Quasi-Z-Source DC-DC Converter With a Novel ZVS and ZCS Technique. IEEE Transactions on Industrial Electronics. 2015; 62(12): 7547-7556.

[183] Sun D, Ge B, Liang W, Abu-Rub H, Peng FZ. An energy stored quasi Z source cascade multilevel inverter based photovoltaic power generation system. IEEE Transactions on Industrial Electronics. 2015; 62(9): 5458-5467.

[184] Priyaa AS, Seyezhai R, Mathur BL. Design and implementation of cascaded Z-Source Multilevel Inverter. International Conference on Advances in Engineering, Science and Management (ICAESM). 2012: 354-360.

[185] Banaei MR, Dehghanzadeh AR.Single Z source based cascaded transformer multilevel inverter. ${ }^{3} \mathrm{rd}$ Power Electronics and Drive Systems Technology Conference (PEDSTC). 2012: 397-402.

[186] Poh Chiang Loh, Feng Gao, Blaabjerg F. Topological and modulation design of three level $Z$ source inverters. IEEE Transactions on Power Electronics. 2008; 23(5): 2268-2277.

[187] Feng Gao, Poh Chiang Loh, Blaabjerg F, Vilathgamuwa D M. Dual Z source inverter with three level reduced common mode switching. IEEE Transactions on Industry Applications; 2007.43(6): 15971608.

[188] Gao F, Loh PC, Blaabjerg F, Teodorescu R, Vilathgamuwa D M. Five level Z source diode-clamped inverter. IET Power Electronics. 2010; 3(4): 500-510.

[189] Gao F, Loh PC, Blaabjerg F, Teodorescu R. Modulation schemes of multi phase three level Z source inverters. IEEE Power Electronics Specialists Conference (PESC). 2007: 1905-1911.

[190] Reza Banaei M, Dehghanzadeh AR. A novel Z-source based multilevel inverter for renewable sources fed DVR. First Power Quality Conference. 2010: 1-6.

[191] V Saravanan, Amal Thukku, R Ramanujam, M Arumugam. A modified seven level $H$ bridge $Z$ Source Inverter for Photovoltaic Systems. Proceedings of the IET-Chennai Third International Conference on Sustainable Energy and Intelligent Systems-SEISCON. 2012: 212-218.

[192] Poh Chiang Loh, Blaabjerg F, Chow Pang Wong. Comparative evaluation of pulse width modulation strategies for Z-Source Neutral-Point-Clamped Inverter. IEEE Transactions on Power Electronics. 2007; 22(3): 1005-1013.

[193] Poh Chiang Loh, Sok Wei Lim, Feng Gao, Blaabjerg F. Three-Level Z-Source Inverters Using a Single LC Impedance Network. IEEE Transactions on Power Electronics. 2007; 22(2): 706-711.

[194] Ding Li, Feng Gao, Poh Chiang Loh, Miao Zhu, Blaabjerg F. Cascaded impedance networks for NPC inverter. Conference Proceedings of IPEC. 2010: 1176-1180. 
[195] Mo Wei, Loh Poh Chiang, Andrew, A, Blaabjerg, F.Single stage buck-boost DC-AC neutral point clamped inverter. IEEE Energy Conversion Congress and Exposition (ECCE). 2012: 318-323.

[196] Poh Chiang Loh, Feng Gao, Blaabjerg F, Shi Yun,Charmaine Feng, Kong Ngai Jamies Soon. Pulse width-Modulated Z-Source Neutral-Point-Clamped Inverter. IEEE Transactions on Industry Applications. 2007; 43(5): 1295-1308.

[197] Mo Wei, Loh Poh Chiang, Andrew, Frede, Blaabjerg.Model Predictive Control of Z-source Neutral Point Clamped Inverter. Energy Conversion Congress and Exposition. 2011: 3838-3843.

[198] Dongsen Sun, Baoming Ge, Xingyu Yan, Daqiang Bi, Hao Zhang, Yushan Liu, Haitham Abu-Rub, Lazhar Ben-Brahim, Fang Zheng Peng. Modeling, Impedance Design, and Efficiency Analysis of Quasi-Z Source Module in Cascaded Multilevel Photovoltaic Power System. IEEE Transactions on Industrial Electronics. 2014; 61(11): 6108-6117.

[199] Mohsen Aleenejad, Hamid Mahmoudi, Reza Ahmadi. A fault-tolerant strategy based on fundamental phase-shift compensation for three-phase multilevel converters with quasi-Z-source networks with discontinuous input current. IEEE Transactions on Power Electronics. 2016; 31(11): 7480-7488.

[200] Yushan Liu, Baoming Ge, Abu-Rub H. Modelling and controller design of quasi-Z-source cascaded multilevel inverter-based three-phase grid-tie photovoltaic power system. IET Renewable Power Generation. 2014; 8(8): 925-936.

[201] Yushan Liu, BaomingGe, Haitham Abu-Rub, Fang Z Peng. An Effective Control Method for QuasiZ-Source Cascade Multilevel Inverter-Based Grid-Tie Single-Phase Photovoltaic Power System. IEEE Transactions on Industrial Informatics. 2014; 10(1): 399-407.

[202] Yushan Liu, Baoming Ge, Haitham Abu-Rub, Fang Zheng Peng. An Effective Control Method for Three-Phase Quasi-Z-Source Cascaded Multilevel Inverter Based Grid-Tie Photovoltaic Power System. IEEE Transactions on Industrial Electronics. 2014; 61(12): 6794-6802.

[203] Liu Y, Ge B, Abu Rub H, Peng F Z. Phase-shifted pulse-width-amplitude modulation for quasi-Zsource cascade multilevel inverter-based photovoltaic power system.IET Power Electronics. 2014; 7(6): 1444-1456.

[204] SM Dehghan, E Seifi, M Mohamadian, R Gharehkhani.Grid connected DG systems based on Z source NPC inverters. ${ }^{2}$ nd Power Electronics, Drive systems and Technologies Conference Proceedings. 2011: 104-110.

[205] Effah FB, Wheeler P, Clare J, Watson A. Space vector modulated three level inverters with a single Z source network. IEEE Transactions on Power Electronics. 2013; 28(6): 2806-2815.

[206] Stepenko S Husev O, Vinnikov D, Ivanets S. FPGA control of the neutral point clamped quasi-Zsource inverter. ${ }^{13}$ th Biennial Baltic Electronics Conference (BEC). 2012: 263-266.

[207] Mo W, Loh PC, Li D, Blaabjerg F. Trans-Z-source Neutral Point Clamped inverter. ${ }^{6}$ th IET International Conference on Power Electronics, Machines and Drives (PEMD). 2012: 1-5.

[208] Ding Li, Poh Chiang Loh, Miao Zhu, Feng Gao, Frede Blaabjerg. Cascaded Trans-Z-source Inverters. ${ }^{8}$ th International Conference on Power Electronics ECCE Asia. 2011: 1976-1980.

[209] Nguyen, Minh Khai, Lim Y, Park S. Improved Trans Z source inverter with continuous input current and boost inversion capability. IEEE Transactions on Power Electronics. 2013; 28(10): 4500-4510.

[210] Ding Li, Poh Chiang Loh, Miao Zhu, Feng Gao, Blaabjerg F. Multi-cell trans-Z-source inverters. IEEE Ninth International Conference on Power Electronics and Drive Systems (PEDS). 2011: 267271.

[211] Ding Li, Poh Chiang Loh, Miao Zhu, Feng Gao, Frede Blaabjerg.Cascaded Multicell Trans Z Source Inverters. IEEE Transactions on Power Electronics. 2013; 28(2): 826-836.

[212] Saman Dadjo Tavakoli; Jasem Khajesalehi; Mohsen Hamzeh, Keyhan Sheshyekani. Decentralized voltage balancing in bipolar dc microgrids equipped with trans-z-source interlinking converter. IET Renewable Power Generation. 2016; 10(5): 703-712.

[213] Aleem Z, Dongsul Shin, Honnyong Cha, Jong-Pil Lee, Dong-Wook Yoo. Parallel operation of inverter using trans-Z-source network. IET Power Electronics. 2015; 8(11): 2176-2183.

[214] Dehnavi SMD, Mohamadian M, Yazdian A, Ashrafzadeh F. Space Vectors Modulation for NineSwitch Converters. IEEE Transactions on Power Electronics. 2010; 25(6): 1488-1496.

[215] Dehghan SM, Mohamadian M, Yazdian A, Ashrafzadeh F. A Dual Input Dual Output Z Source Inverter. IEEE Transactions on Power Electronics. 2010; 25(2): 360-368.

[216] Azizi M, Fatemi A, Mohamadian M, Yazdian A. A novel Z source four leg inverter with two independent four wire outputs. Power Electronic \& Drive Systems \& Technologies Conference (PEDSTC). 2010: 163-168.

[217] Dehghan SM, Mohamadian M, Yazdian A, Ashrafzadeh F. A novel space vector modulation for nine switch converters. Energy Conversion Congress and Exposition (ECCE).2009: 885-891.

[218] Dehghan SM, Mohamadian M, Yazdian A. Hybrid Electric Vehicle Based on Bidirectional Z-Source Nine-Switch Inverter. IEEE Transactions on Vehicular Technology. 2010; 59(6): 2641-2653.

[219] Dehghan, SM, Mohamadian M, Rajaei AH, Yazdian A. Full modeling a series HEV based on BZSNSI. ${ }^{2}$ nd Power Electronics, Drive Systems and Technologies Conference (PEDSTC). 2011: 439444. 
[220] Dong Cao, Shuai Jiang, Xianhao Yu, Fang Zheng Peng. Low cost semi-Z-source inverter for single phase photovoltaic systems. IEEE Transactions on Power Electronics. 2011; 26(12): 3514-3523.

[221] Ahmed T, Mekhilef S. Semi Z source inverter topology for grid connected photovoltaic system. IET Power Electronics. 2015; 8(1): 63 -75.

[222] Marek Adamowicz. LCCT-Z-Source Inverters. ${ }^{10}$ th International Conference on Environment and Electrical Engineering (EEE IC). 2011: 1-6.

[223] Adamowic M, Strzelecki R, Fang Zheng Peng, Guzinski J, Rub HA. New Type LCCT-Z-Source Inverters. Proceedings of the ${ }^{14}$ th European Conference on Power Electronics and Applications (EPE 2011). 2011: 1- 10.

[224] Marek Adamowicz, Jaroslaw Guzinski, Ryszard Strzelecki, Fang Zheng Peng, Haitham Abu-Rub. High step-up continuous input current LCCT-Z source inverters for fuel cells. Energy Conversion Congress and Exposition. 2011: 2276-2282.

[225] Strzelecki R, Bury W, Adamowicz M Strzelecka N. New alternative passive networks to improve the range output voltage regulation of the PWM inverters. Twenty-Fourth Annual IEEE conferences Applied Power Electronics Conference and Exposition (APEC). 2009: 857-863.

[226] Minh Khai Nguyen, Young Cheol Lim, Sung Jun Park, Young Gook Jung. Cascaded T Z source inverters. IET Power Electronics. 2014; 7(8): 2068-2080.

[227] Jing Jun Soon, Kay Soon Low. Sigma Z source inverters. IET Power Electronics. 2015; 8(5): 715723.

[228] Fathi H, Madadi H. Enhanced Boost Z Source Inverters With Switched Z Impedance. IEEE Transactions on Industrial Electronics. 2016; 63(2): 691-703.

[229] Loh Poh Chiang, Li Ding, Blaabjerg Frede. Current-type flipped- $\Gamma$-source inverters. ${ }^{7}$ th International Power Electronics and Motion Control Conference (IPEMC). 2012: 594-598.

[230] Poh Chiang Loh, Ding Li, Blaabjerg F. Voltage-type $\Gamma$-source inverters. ${ }^{7}$ th IEEE Conference on Industrial Electronics and Applications (ICIEA). 2012: 781-786. 